\title{
No linealidad del comportamiento elástico de las cerámicas piezoeléctricas
}

\author{
A. ALBAREDA, R. PÉREZ, J.E. GARCÍA, J.A. CASALS, M. UDINA \\ Dep. Física Aplicada. Univ. Politécnica de Catalunya. Campus Nord Ed. B4. Barcelona
}

\begin{abstract}
Se describen y comparan tres métodos experimentales de la caracterización no lineal del comportamiento elástico de resonadores, según se mantenga constante la tensión, la corriente o la frecuencia. Se muestra la equivalencia entre los tres métodos, describiendo con detalle la implementación del último. La alteración de la impedancia eléctrica en función de la corriente en el entorno de la frecuencia de resonancia es la mejor forma de expresar el comportamiento no lineal, permitiendo predecir la aparición de histéresis. La relación entre incrementos no lineales de la reactancia $X$ y de la resistencia $R$ se mantiene notablemente lineal, la dependencia de estas magnitudes con la corriente mecánica obedece a una ley potencial de exponente no entero. Para evitar el calentamiento inherente a las medidas con alta señal se propone la excitación por medio de trenes de onda o ráfagas, y el tratamiento posterior de la respuesta eléctrica y de la velocidad $v$, obtenidas durante el régimen estacionario. El método se aplica adecuadamente tanto a cerámicas PZT blandas como duras. Sus características elásticas se comparan con las características dieléctricas y piezoeléctricas no lineales, obteniendo una relación explicable por el predominio de los mecanismos extrínsecos sobre los intrínsecos.
\end{abstract}

Palabras clave: Piezoeléctricos, cerámicas, no linealidad, coeficiente elástico, dieléctrico.

\section{Nonlinear elastic behaviour of piezoelectric ceramics}

Three experimental methods of nonlinear characterization of elastic behaviour are compared, based upon constant voltage, constant current and constant frequency respectively. Equivalence between them is shown, and implementation of the last one is described in detail.

The best way to describe nonlinear behaviour is to look at the alteration of the electrical impedance as a function of the current level, around the resonance frequency. By taking this description, we are able to compute the onset of hysteresis phenomena. Between the nonlinear increment of the reactance $X$ and the resistance $R$ appears a fairly linear relationship, while the quantities of $X$ and $\mathrm{R}$ depend on the motional current through a fractional power function. In order to avoid the overheating inherent to high signal measurement, an excitation by bursts is proposed, and electrical response and velocity, obtained during the steady state, has been treated afterwards. This method has been applied to hard and soft PZT ceramics. Elastic electrical and piezoelectric behaviours have been compared, leading to some relations that can be explained by the predominance of the extrinsic processes over the intrinsic onaes.

Key words: Piezoelectricity, ceramics, non linearity, elastic coefficient, dielectric.

\section{INTRODUCCIÓN}

Debido al gran valor de sus coeficientes piezoeléctricos, y a su buen factor de acoplo electromecánico, las cerámicas de tipo perovskita, como el Titanato Zirconato de Plomo (PZT), son casi insustituibles en algunas aplicaciones en las que se requiere gran respuesta mecánica (como en los actuadores) o bien alta potencia (como en los transductores de ultrasonidos) $(1,2)$.

Estas cualidades, cuyo origen debe buscarse en la particular estructura de granos y de dominios ferroeléctricos de dichos materiales, contrastan con algunos inconvenientes inherentes a dicha estructura (3): pérdidas energéticas elevadas, dependencia notable con la temperatura, poca estabilidad a lo largo del tiempo, aparición de fatiga y elevada no linealidad de su comportamiento (4).

En una cerámica, la mayor parte de los efectos dieléctricos y piezoeléctricos no proviene de la deformación de las celdas cristalinas por aplicación de campo eléctrico o de esfuerzo mecánico (efecto intrínseco), sino del movimiento de las paredes de dominio, especialmente de las que separan dominios de polarización no totalmente opuesta (distintas de $180^{\circ}$ ) (efecto extrínseco) $(5,6,7)$.

Esta estructura, que debe alterarse profundamente en el proceso de polarización de la cerámica, necesario para romper la simetría y con ello pueda tener propiedades piezoeléctricas, es poco estable, sufriendo relajaciones de diversa índole, que se reflejan en el envejecimiento y la fatiga del material.

Debido a que el efecto piezoeléctrico es pequeño, es frecuente que en un dispositivo se tenga que aplicar esfuerzos mecánicos o campos

eléctricos cercanos al límite, por lo que resulta conveniente para el diseño de dispositivos conocer la degradación que sufre al acercarnos a dichas condiciones. Por otra parte, el esclarecimiento de los mecanismos involucrados en dichos procesos puede permitir la obtención de nuevos materiales mejor adaptados a un uso específico.

La aplicación de campos y esfuerzos elevados conlleva la aparición de dos tipos de efectos: en primer lugar, si la potencia disipada en el material es elevada, y se aplica durante un tiempo largo, se puede producir un sobrecalentamiento que puede alterar sus características. En segundo lugar, la simple aplicación de esos campos, aun cuando sea de corta duración y no se produzca calentamiento, produce fenómenos ligados al comportamiento no lineal $(8,9,10)$.

Los fenómenos que aparecen al aplicar un campo elevado (continuo o alterno) son:

1. Alteración de los coeficientes característicos del material, que pueden ser de tipo dieléctrico, elástico o piezoeléctrico.

2. Aumento de las pérdidas, lo que puede empeorar los problemas de tipo térmico.

3. La generación de armónicos, apareciendo señales de frecuencia distinta a la aplicada.

4. Aparición de histéresis, ya sea en la aplicación de campos casi estáticos, como en la aplicación de frecuencias en el entorno de las resonancias mecánicas.

Dichos efectos se observaran o no según las circunstancias de trabajo. Si se aplican campos eléctricos a baja frecuencia, estos deben 
ser elevados para obtener deformaciones importantes, con lo cual aparecen no linealidades fundamentalmente dieléctricas. En cambio, si se hace trabajar en resonancia, para conseguir la misma deformación el campo aplicado puede ser mucho menor, y las no linealidades aparecen como consecuencia de los grandes esfuerzos y a las grandes deformaciones que sufre el material.

Una de las primeras dificultades que se tienen al intentar describir el comportamiento no lineal, consiste en determinar cuales son las magnitudes que convienen medir, y con qué magnitudes se deben relacionar. Un ejemplo de ello es el hecho de que si bien las primeras medidas de la no linealidad en la resonancia se realizaban en función de la tensión aplicada, posteriormente se ha medido en función de la corriente, lo que ha supuesto una simplificación notable de las funciones que describen su comportamiento.

Por último, en contra de lo que ocurre en las medidas lineales, la amplitud de la excitación no es arbitraria. Conviene explorar el comportamiento del material hasta situaciones límite. Por ello hay que prevenir el sobrecalentamiento, a fin de no mezclar los efectos no lineales con los efectos térmicos.

La medida de la no linealidad elástica se puede llevar a término en condiciones estáticas. Pero puede ser conveniente realizar la medida aprovechando la resonancia, sobre todo si se pretende usar el material en un dispositivo resonante. Una ventaja obvia de este método es que la medida se realiza de forma totalmente eléctrica. Por otro lado, el campo eléctrico a aplicar suele ser muy pequeño, por lo que se minimizan la no linealidad dieléctrica que podrían interferir en la medida. En contrapartida, la medida solo se puede realizar a una frecuencia determinada, dependiente del tamaño del resonador.

\section{FENÓMENOS NO LINEALES EN LA RESONANCIA}

Cuando se aplica alta señal a un resonador, se observan en el entorno de la resonancia, un aumento de las pérdidas mecánicas, una disminución de la frecuencia de resonancia y aparece histéresis frecuencial al hacer barridos de frecuencia a tensión constante. Estos tres fenómenos (9) dependen de las características de los materiales, siendo más acusado el comportamiento no lineal en los materiales piezoeléctricos blandos que en los duros. En general, afectan a los dispositivos resonantes, disminuyendo su potencia y eficiencia o bien, complicando el amplificador (driver) que sirve de excitación al resonador.

Cuando se aplica una señal pequeña a un resonador, su comportamiento eléctrico puede describirse mediante un circuito equivalente, consistente en una rama eléctrica (formada por un condensador $C_{0}$ ) en paralelo con una rama mecánica, de impedancia mecánica $Z_{1}$ (formada por un circuito $R_{1} L_{1} C_{1}$ en serie). En el entorno de la frecuencia de resonancia de la rama mecánica, la resistencia total $R_{1}$ apenas se altera al cambiar la frecuencia, mientras que la reactancia $X_{1}$ es lineal con ella. Ello significa que en el plano de la admitancia $Y$ se dibuja un círculo casi perfecto. Barriendo en frecuencia, al aplicar una tensión constante, se produce un pico de corriente que es proporcional a la tensión aplicada (según el comportamiento lineal) y cuya anchura depende del factor de calidad.

La aplicación de una tensión cada vez mas elevada produce (Fig. 1), por un lado, que no se mantenga la proporcionalidad (la admitancia $Y$ máxima disminuye, aumentando las pérdidas). Por otro lado, el pico se desplaza hacia frecuencias inferiores, a la vez que se ensancha y deja de ser simétrico $(9,11)$. Si el efecto es suficientemente intenso, el pico puede desplazarse un tramo mayor que su anchura, produciéndose un fenómeno de histéresis, con lo que aparecen dos respuestas estables distintas para una determinada excitación: la admitancia difiere si el barrido de frecuencias se realiza con frecuencias crecientes o decrecientes. Sólo si el barrido es decreciente se alcanza el máximo de admitancia, es decir, la máxima potencia del resonador.

A estos tres fenómenos debemos añadir la producción de armónicos o subarmónicos de la frecuencia de excitación, aunque dicho fenómeno no se tratará a lo largo de este trabajo.

\subsection{Estudio en el plano de impedancias.}

Para comprender mejor los fenómenos no lineales que ocurren en la resonancia proponemos un estudio en el plano de impedancias "mecánicas" (o motional) $Z_{1^{\prime}}$ del circuito equivalente anteriormente citado. Se observa experimentalmente que al aumentar la tensión del generador $V_{g}$ la curva $Z_{1}(\omega)$ se deforma (Fig. 2), pasando de ser una recta, con la parte resistiva constante para baja señal, a deformarse en la proximidad de la resonancia, para alta señal. Tanto la parte real $R_{1}$ como la reactancia $X_{1}$ aumentan con la tensión $V_{g}$ para una misma fre-

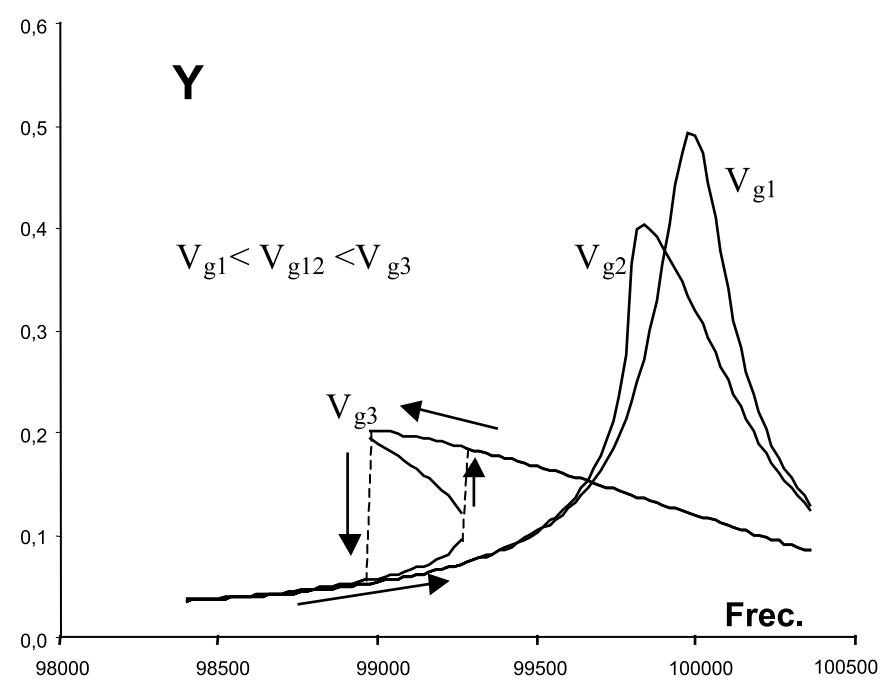

Fig. 1: Admitancia de un resonador en las proximidades de la resonancia y para valores de la tensión de generador crecientes.

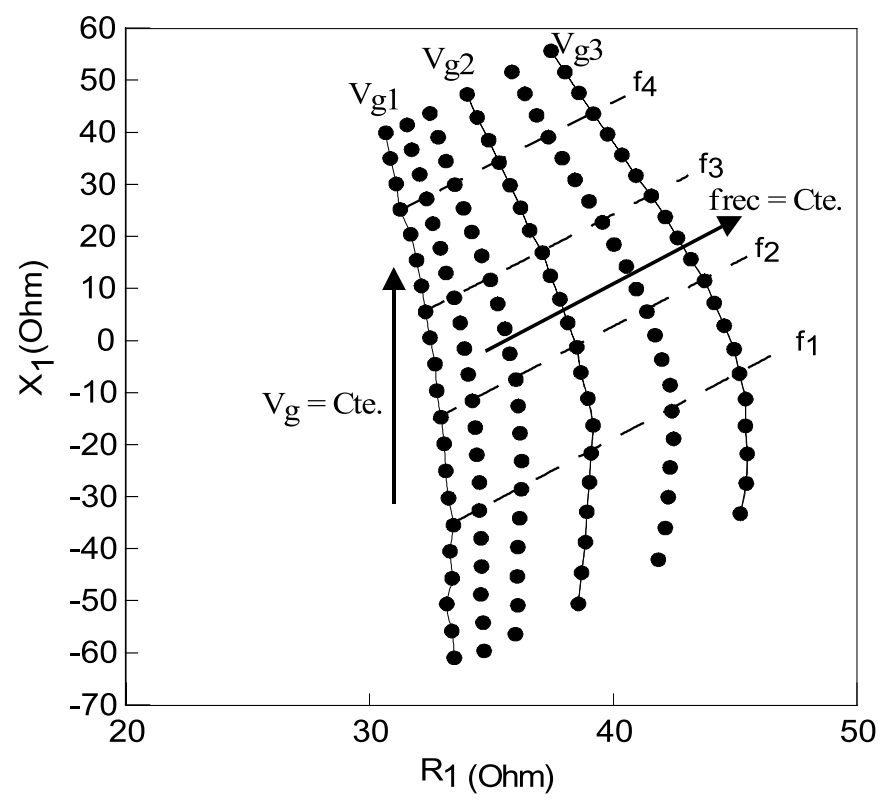

Fig. 2: Impedancia mecánica de un resonador en las proximidades de la resonancia. Curvas a tensión de generador constante y a frecuencia constante. 
cuencia. Existe una equivalencia entre el aumento de las pérdidas mecánicas y el incremento de la resistencia de la rama "mecánica" $R_{1}$ del circuito equivalente del resonador. También hay una relación directa entre la disminución de la frecuencia de resonancia $\Delta f_{s}$ y el aumento de la reactancia "mecánica" $\Delta X_{N L}$. Efectivamente, para alcanzar la resonancia $X_{1}=0$ con alta señal es preciso aplicar una frecuencia menor que la frecuencia de resonancia a baja señal $f_{s}<f_{s 0}(11)$ :

$$
\begin{aligned}
& Z_{1}=Z_{10}+\Delta Z_{N L}=R_{10}+\Delta R_{N L}+j\left(L_{10} \omega-\frac{1}{C_{10} \omega}\right)+j \Delta X_{N L} \\
& \tan \delta_{m}=\frac{R_{1}}{\bar{Z}}=\frac{R_{10}+\Delta R_{N L}}{\bar{Z}}, \frac{\Delta f_{s}}{f_{s 0}}=\frac{f_{s}-f_{s 0}}{f_{s 0}}=-\frac{\Delta X_{N L}}{2 \bar{Z}} \\
& \text { donde } \quad \bar{Z}=\frac{1}{C_{10} \omega_{s 0}} .
\end{aligned}
$$

Se observa experimentalmente en el plano de impedancias (Fig. 2) que la variación no lineal de impedancia $\Delta Z_{N L}$ mantiene su argumento aunque varíe la amplitud de la excitación y de la no linealidad. En efecto, el cociente $\Delta X_{N L} / \Delta R_{N L}=m$ se mantiene casi constante si únicamente varia la amplitud de la excitación, pero se mantiene constante la frecuencia.

Las dependencias entre las partes reales e imaginarias de la impedancia "mecánica" con la corriente "mecánica" $R_{1}\left(I_{1}\right)$ y $X_{1}\left(I_{1}\right)$ (Fig. 3) muestran experimentalmente una contribución no lineal que es independiente de la frecuencia, dependen sólo de la amplitud $I_{1}$ de la excitación. Por lo tanto estas dependencias nos proporcionan una herramienta inmejorable para la caracterización no lineal con alta señal. A la vez esta dependencia de las pérdidas y del corrimiento de frecuencia se puede expresar con una buena aproximación como una función potencial de la corriente "mecánica". Proponemos el uso de esta corriente mecánica $I_{1}$ como variable independiente macroscópica, pues al ser proporcional a las grandes deformaciones $S$ que se producen en la resonancia, es la magnitud que rige las no linealidades.

Para caracterizar correctamente al material (y no al dispositivo) es preciso que las magnitudes a relacionar no dependan de las dimensiones del resonador. Para ello se buscarán las leyes que rigen la no linealidad en función de la parte mecánica del desplazamiento eléctrico $D_{1^{\prime}}$ o bien de la deformación elástica media $\langle S\rangle$, en lugar de la corriente mecánica $I_{1}$ :

$$
\begin{aligned}
& \Delta \tan \delta_{m}=\rho D_{1}{ }^{n} \quad, \quad \frac{|\Delta \mathrm{c}|}{\mathrm{c}_{0}}=2 \frac{\Delta f_{s}}{f_{s 0}}=\chi D_{1}{ }^{n} \\
& \text { donde }<\mathrm{S}>=\frac{\mathrm{D}_{1}}{\sqrt{M}}=\frac{\mathrm{I}_{1}}{\mathrm{~A} \omega_{\mathrm{s}} \sqrt{M}},
\end{aligned}
$$

siendo $A$, el área de los electrodos y $M$ es un factor de mérito electromecánico del resonador, que varía en función del modo de vibración y del coeficiente piezoeléctrico correspondiente.

La potencia $n$ asociada a estas funciones depende del material, mostrándose experimentalmente que su valor es próximo a la unidad en el caso de cerámicas piezoeléctricas blandas, mientras que en las cerámicas duras tienen valores próximos a 2 (Tabla I) (12).

En el plano de impedancias puede comprenderse bien el fenómeno de histéresis frecuencial que se produce en la curva $Y(\omega)$ con alta señal (Fig. 4): cuando la tensión de excitación $V_{g}$ constante se hace grande, puede ocurrir que las rectas de pendiente $m$ a frecuencia constante corten a las curvas $Z_{1}(\omega)$ no en 1 punto (como ocurre a baja o media señal, punto A) sino en 3 puntos (para $3 V_{\text {y }} f_{1}<f<f_{2}$ ). En este caso, de estos 3 puntos, 2 son estables y el intermedio es inestable e inalcanzable. Se alcanza uno u otros de los puntos estables dependiendo de las condiciones anteriores; por ejemplo, dependiendo de si el barrido de frecuencias es con frecuencias crecientes (puntos entre B
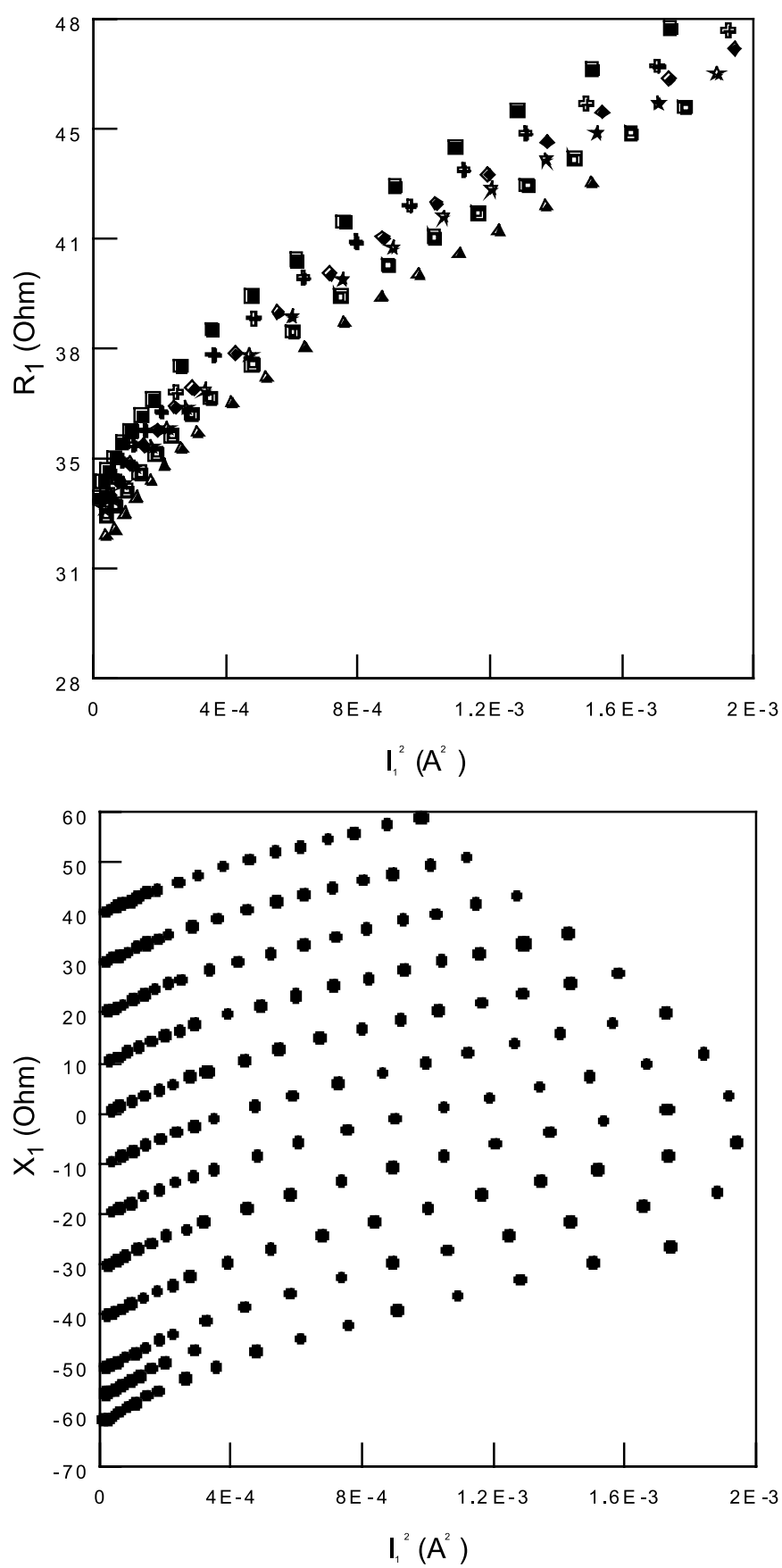

Fig. 3: Dependencia de la resistencia mecánica (a) y de la reactancia mecánica (b) con la corriente mecánica, para diversas frecuencias próximas a la resonancia..

y C) o decrecientes (puntos entre E y D). Esto se ha observado (11), mostrándose una zona en el plano de impedancias donde no aparecen puntos experimentales.

Esta observación nos permite analizar cuando se puede producir la histéresis: cuando la pendiente de la curva $Z_{1}(\omega)$, es decir $d X_{1} / d R_{1}$ sea menor que la pendiente $m$ de las rectas a frecuencia constante:

$$
\frac{d X_{1}}{d R_{1}}<\frac{\Delta X_{N L}}{\Delta R_{N L}}=m
$$

Es de señalar que estas pendientes son una magnitud característica del material, que vale aproximadamente $m=2$, para los materiales 


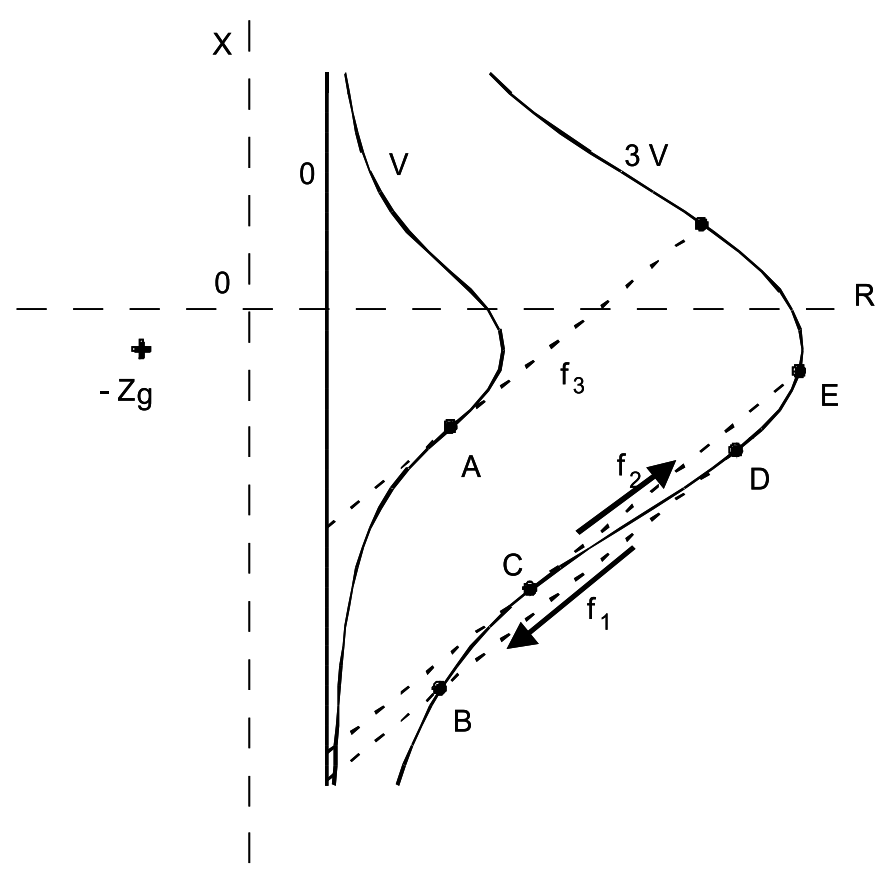

Fig. 4: Visualización del fenómeno de histéresis en el plano de impedancias.

blandos, mientras que para las cerámicas duras tiene un valor más elevado, entre 6 y 15 (ver Tabla I). Por lo tanto, será más frecuente encontrar el fenómeno de histéresis en los materiales duros, puesto que en ellos la desigualdad (Ec. 4) es más fácil que se produzca.

TABLA I: VALORES DE LOS COEFICIENTES Y EXPONENTES NO LINEALES MECÁNICOS Y DIELÉCTRICOS, PARA DOS TIPOS DE CERÁMICAS PZT.

\begin{tabular}{|c|ccccc|cccc|}
\hline material & $\mathrm{Q}_{\mathrm{m}}$ & $\mathrm{n}$ & $\chi$ & $\rho$ & $\mathrm{m}$ & $\varepsilon^{\prime}(0)$ & $\tan \delta_{\mathrm{E}}$ & $\eta$ & $\Delta \varepsilon^{\prime} / \Delta \varepsilon^{\prime \prime}$ \\
& & & $.10^{-3}$ & $.10^{-3}$ & & & $.10^{-3}$ & & \\
\hline pz27 & 90 & 0,65 & 83 & 26 & 3,1 & 1677 & 23 & 0,9 & 2,3 \\
pz26 & 2900 & 1,94 & 6,1 & 0,8 & 7,8 & 1424 & 4,3 & 2,1 & 9,5 \\
\hline
\end{tabular}

Este fenómeno de histéresis depende también de la impedancia del generador, pues la contribución no lineal es función de la corriente que circula (11). Esto significa que puede eludirse la histéresis si se utiliza un generador con una impedancia interna suficientemente elevada, como en el caso de un generador de corriente.

\section{CARACTERIZACIÓN NO LINEAL}

La caracterización no lineal se ha realizado clásicamente como una extensión de la caracterización lineal de los materiales y dispositivos resonantes, es decir, con analizadores de impedancias o analizadores de redes o vectoriales.

\subsection{Barridos de frecuencia, con tensión de generador constante.}

Es la forma como clásicamente se ha llevado a cabo la caracterización no lineal (8). Los estándares europeos así lo contemplan (9). Las medidas se realizan con barridos de frecuencia decreciente para que, en el caso de que ocurra la histéresis frecuencial, se consiga alcanzar el máximo de admitancia a la frecuencia de resonancia. En este caso se debe medir la frecuencia de resonancia $f_{s}$ (admitancia máxima) y las pérdidas mecánicas (resistencia $R$ ) así como la corriente $I$. De estos valores se obtienen los parámetros intrínsecos a los materiales, es decir la variación relativa de la frecuencia de resonancia y la tangente de pérdidas mecánicas, magnitudes que se representan en función del desplazamiento medio $\langle S\rangle$, proporcional a la corriente mecánica o motional $I_{1}$ (Ec. 3) (Tabla I).

En este tipo de barrido a tensión de generador constante, la potencia que se disipa en el resonador, y que es máxima en la resonancia, vale:

$$
\mathrm{P}_{\mathrm{dis}}=\frac{\mathrm{RV}_{\mathrm{g}}^{2}}{Z^{2}}
$$

por lo que, a excepción de algunos pocos puntos a frecuencias muy próximas a la resonancia, donde la potencia es elevada, en general, la impedancia total $Z$ será elevada y por lo tanto esta potencia disipada será pequeña.

\subsection{Barridos de frecuencia, con corriente mecánica constante.}

En este tipo de barridos, propuesto por Hirose (13), se debe utilizar un generador de corriente, adaptando dicha corriente constante al valor de la corriente mecánica $I_{1}$. Las medidas que se efectúan son, la frecuencia de resonancia (mínima tensión) y valor de esta tensión para cada $I_{1}$. De estos valores fácilmente se obtienen las leyes de la variación no lineal de las pérdidas y del corrimiento de frecuencia.

La ventaja principal de este método de medida es que evita la histéresis, e incluso obtiene curvas de resonancia simétricas, ambas cosas no ocurren con el barrido con tensión constante. Este resultado es perfectamente explicable desde el punto de vista del plano de impedancias. Al llevar a cabo un barrido con $I_{1}$ constante, también las variaciones no lineales de impedancia mecánica son constantes en todo el barrido. Al aumentar la corriente $I_{1}$ la gráfica de $Z(\omega)$, de la impedancia del resonador al variar la frecuencia, simplemente se ha desplazado oblicuamente (según el valor constante $\Delta Z_{N L}$ ), manteniéndose rectas, pero no se ha deformado haciendo la curvatura que se mostraba en la gráfica 2. Por lo tanto, al no haber esta curvatura los puntos de intersección entre las rectas citadas a $I_{1}$ constante con las rectas a frecuencia constante no dan lugar a soluciones inestables, evitando la histéresis, ni a asimetrías antes y después de la resonancia.

Sin embargo este sistema de medida presenta algunos inconvenientes: utiliza un generador no convencional, a corriente mecánica constante, y la potencia aplicada es más elevada. En efecto la potencia disipada en este tipo de barrido es $I^{2} R$ que es casi constante e igual a la potencia máxima que se disipa en el barrido de frecuencias con tensión constante.

\subsection{Barridos de amplitud, a frecuencia constante.}

El uso de un barrido de amplitudes a frecuencia constante, tiene la gran ventaja de que con un único barrido se realiza la caracterización total del resonador. Se trata de medir la parte real $R$ e imaginaria $X$ de la impedancia $Z$ del resonador, así como la corriente $I$ que atraviesa el resonador, para una única frecuencia próxima a la frecuencia de resonancia y para amplitudes crecientes (11). Para ser más precisos interesa colocarse a una frecuencia $f$ que corresponda aproximadamente a la frecuencia de resonancia con la máxima amplitud $f s$ que se utiliza. Para ello la frecuencia de barrido debe ser menor que la frecuencia de resonancia a baja señal $f<f_{s 0^{\circ}}$. De los datos anteriores, y gracias a las anteriores expresiones Eq. 2-3, se puede obtener las dependencias no lineales, de la tangente de pérdidas y de la variación de la frecuencia de resonancia, en función de la contribución mecánica al desplazamiento eléctrico $D_{1}$. 
Para confirmar que las leyes que se obtengan son independientes de la frecuencia, tal como se afirma en el anterior apartado 2.1, se realizan otros barridos de amplitudes a otras frecuencias menores y mayores a la propuesta. Del análisis de las leyes $\tan \left(\delta_{m}\right)\left(D_{1}\right)$ y $\Delta f / f_{s 0}$ $\left(D_{1}\right)$ a estas frecuencias puede verificarse que las leyes propuestas son independientes de la frecuencia del barrido de amplitudes. Por lo tanto, no es preciso realizar varios barridos de frecuencia con tensiones o corrientes constantes, sino que basta un solo barrido de amplitudes a una única frecuencia.

El principal inconveniente de este sistema de medida es el problema que no todos los analizadores de impedancias o de redes disponen de la posibilidad de realizar barridos de amplitud, mientras que todos estos equipos si que tienen la posibilidad de llevar a cabo barridos de frecuencia.

\section{MEDIDAS CON TRENES DE ONDA O RÁFAGAS}

Un aspecto común a los 3 métodos citados anteriormente es el uso de analizadores de impedancia o de redes como equipo básico de medida. Ello implica a su vez que se está usando una señal sinusoidal continuamente, por lo que se está sobrecalentando la muestra mientras se efectúa la medida. Para evitar el solapamiento de estos dos fenómenos, sobrecalentamiento y comportamiento no lineal, se deben efectuar las medidas en un corto intervalo de tiempo, típicamente en menos de 2 segundos. A pesar de esta precaución las medidas anteriores se ven limitadas a potencias medias. Por ejemplo para la caracterización de discos de unos $2 \mathrm{~cm}$ de diámetro y $1 \mathrm{~mm}$ de grosor la potencia máxima alcanzada en esta caracterización es de unos $4 \mathrm{~W}$.

Una posibilidad para evitar el sobrecalentamiento en las medidas no lineales de potencia es utilizar trenes de onda en vez de señales sinusoidales en forma continuada (14). Los trenes de onda se generan con la amplitud y frecuencia deseada (próxima a la de resonancia) y se repiten lentamente entre ellas para evitar ese sobrecalentamiento (frecuencia de repetición de $10 \mathrm{~Hz}$ ). Con ese sistema solo se excita al resonador una pequeña fracción de tiempo, por ejemplo entre el $1 \%$ y el 3\%. Sin embargo este tipo de excitación invalida el uso de los analizadores de impedancias y debe registrarse la corriente de respuesta en función del tiempo (por ejemplo, mediante un osciloscopio) (Fig. 5). Una ventaja de este sistema es la similitud de esta excitación con muchas de las aplicaciones de potencia de los resonadores.

\subsection{Medidas en el régimen transitorio}

En un primer momento se utilizó estos trenes de onda y se llevó a cabo la medida de la respuesta transitoria en la cola de la respuesta del resonador. En la gráfica $I(t)$ de la cola del transitorio se medía, para diferentes instantes $t_{i^{\prime}}$ la frecuencia de la señal y la constante de tiempo $\tau$ de la envolvente, para con estos datos, y con los filtros y tratamientos digitales más adecuados, obtener las leyes de variación de las pérdidas y variación de la frecuencia de resonancia en función de la amplitud de la corriente en ese instante $t_{i}$ :

$$
\tan \left(\delta_{\mathrm{m}}\right)=\frac{1}{Q}=\frac{1}{\omega_{\mathrm{s}} \tau}
$$

Se trata en este caso de asumir que las oscilaciones amortiguadas que se observan tienen una amplitud o envolvente que no cumple exactamente una ley exponencial como sería de esperar de un sistema lineal, ni tampoco una frecuencia constante. Sin embargo se puede aceptar que el sistema no lineal que se estudia es solo débilmente no lineal. Ello implica que el transitorio lo podemos tratar como una función que es aproximadamente exponencial para cada tramo estudiado,

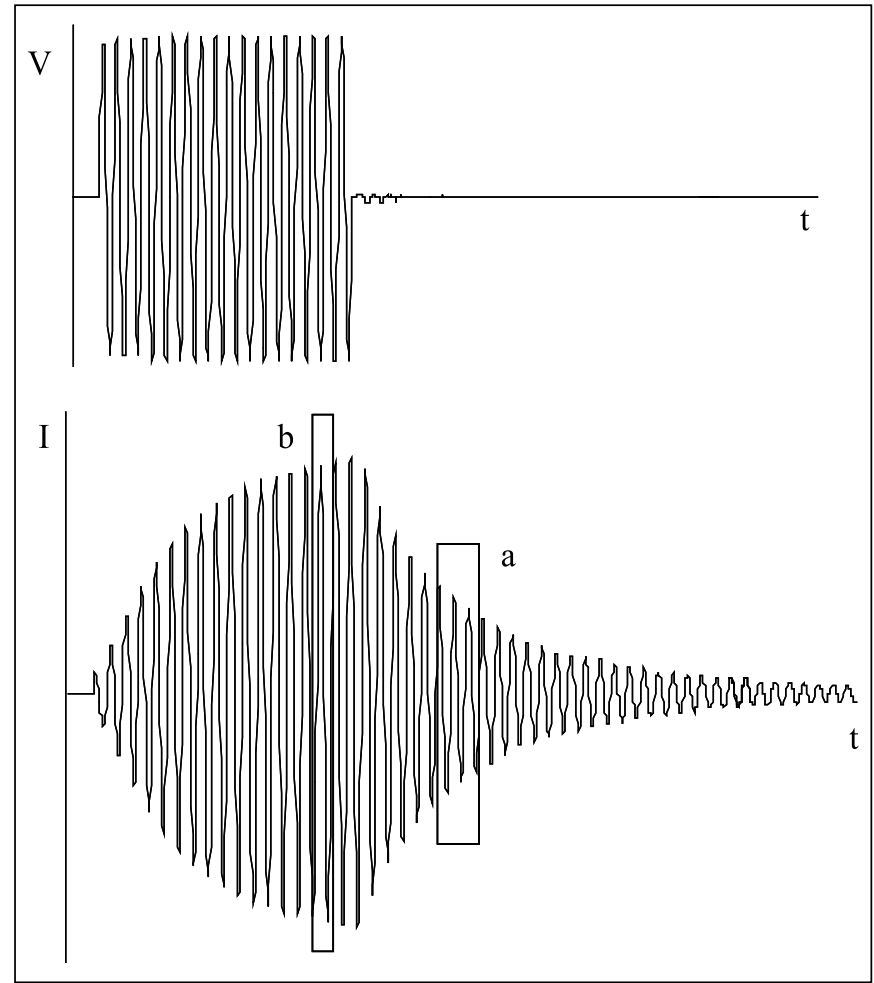

Fig. 5: Tensión de excitación de un tren de onda y corriente de respuesta de un resonador. Se realizan las medidas en el régimen transitorio (a) o bien al final del régimen estacionario (b).

pero con valores de la constante de tiempo $\tau$ y de la frecuencia de oscilación $\omega_{\mathrm{s}}$ que dependen de la amplitud en ese instante $I\left(t_{i}\right)$.

Un método muy similar ha sido descrito en la caracterización no lineal de cerámicas piezoeléctricas con elevado factor de calidad (15). Sin embargo cuando se utilizó este sistema en la caracterización de composites piezoeléctricos 1-3 para ser utilizados en la emisión de ultrasonidos en el aire se observó la dificultad de obtener leyes con suficiente precisión. Este método puede ser bueno siempre y cuando se utilice sólo en el primer modo de vibración, es decir en ausencia de otros modos de vibración próximos.

\subsection{Medidas en el régimen estacionario}

Puesto que con el método de utilizar trenes de onda se han conseguido la caracterización de cerámicas con una excitación mucho mayor (pasando, por ejemplo, de una potencia de $4 \mathrm{~W}$ hasta $200 \mathrm{~W}$, para las muestras citadas), se ha llevado a cabo la caracterización en el régimen estacionario de la respuesta del resonador, en lugar de utilizar el régimen transitorio (Fig. 5). Para ello se utilizan trenes de onda que tengan un número de ciclos $N$ suficientemente elevado para asegurar que el resonador ha alcanzado el régimen estacionario. Gracias a un osciloscopio de memoria se adquieren las señales $V(t)$ así como $I(t)$, y un tratamiento adecuado de estas señales, por un ordenador, permite obtener la impedancia $Z$ para cada amplitud $I$. Basta con repetir el proceso para otras amplitudes para obtener las leyes no lineales deseadas $R(I), X(I)$ y gracias a las expresiones (1-2) obtener las leyes no lineales $\tan \left(\delta_{m}\right)\left(D_{1}\right)$ y $\Delta f / f_{s 0}\left(D_{1}\right)$ que son independientes de los tamaños del resonador.

Con este sistema de medida se obtienen todos los beneficios de la excitación por trenes de onda pero obteniendo las leyes no lineales a la frecuencia deseada, evitando los posibles batidos entre diferentes modos de vibración. 


\section{RESULTADOS OBTENIDOS EN LA CARACTERIZACIÓN MECÁNICA}

En la figura 6 se observa los resultados experimentales obtenidos para un resonador, para diferentes frecuencias próximas a la resonancia. Los círculos que se obtienen en el plano de impedancias $Z$ muestran como al aumentar la reactancia $X$, por la no linealidad, se alcanza la antiresonancia, sin tener que variar la frecuencia. En la figura 7 se ha representado esta misma experiencia pero en el plano de las impedancias "mecánicas" $Z_{1}$, obteniendo unas leyes prácticamente idénticas para la contribución no lineal a las diferentes frecuencias. Se confirma que existe una estrecha relación $\Delta X_{N L} / \Delta R_{N L}=m$ entre las variaciones no lineales de la resistencia y reactancia (11). De la observación de ambas gráficas se deduce que las leyes son más adecuadas cuando se describen en el plano de las impedancias mecánicas.

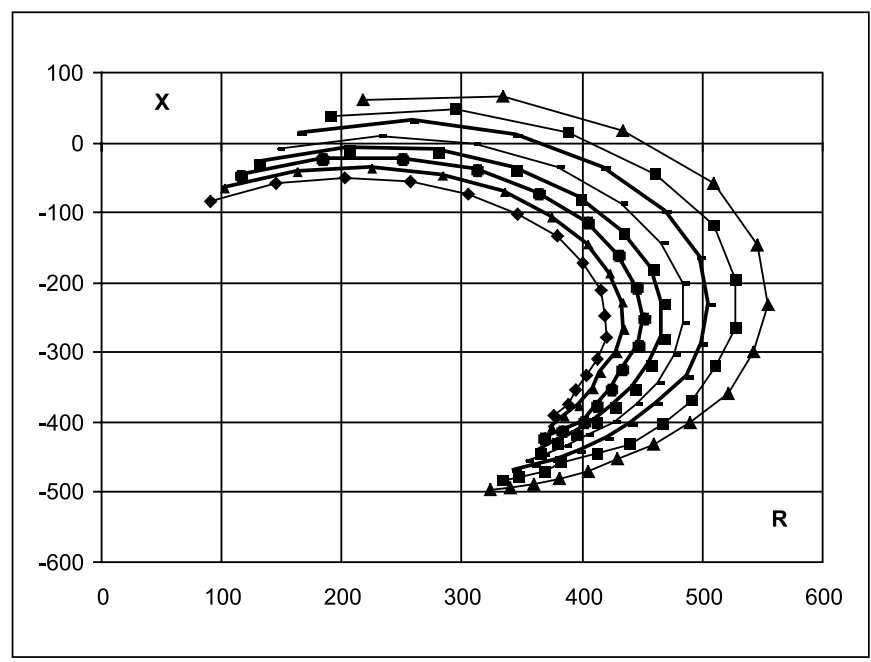

Fig. 6: Resultados obtenidos en el plano de impedancias, al hacer un barrido de amplitudes a frecuencia constante (próxima a la resonancia) utilizando la excitación en trenes de onda.

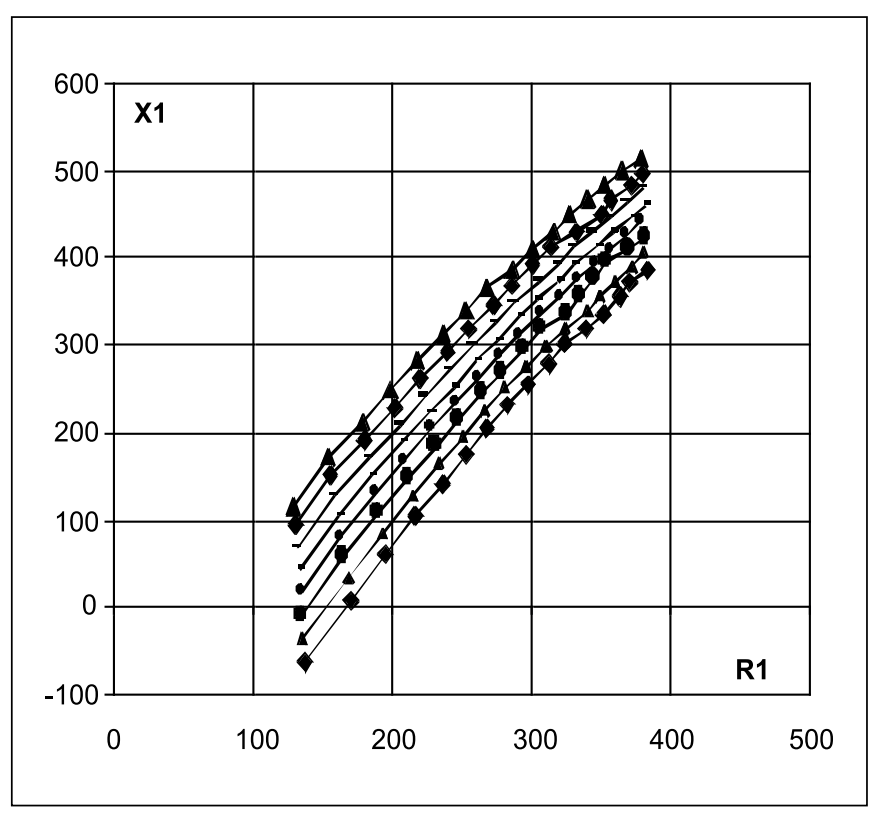

Fig. 7: La misma representación anterior, pero en el plano de las impedancias mecánicas. El comportamiento no lineal es idéntico para las distintas frecuencias.
La figura 8 muestra la dependencia entre la reactancia mecánica y la deformación media, corrigiendo el desplazamiento de la reactancia debido a la variación de la frecuencia a la cual se ha realizado el barrido de amplitudes. En esta gráfica puede comprobarse que la dependencia no lineal depende exclusivamente de la deformación. De estas gráficas se han obtenido los coeficientes que rigen las no linealidades (Ec. 3), tanto para los materiales blandos como para los duros, y que se muestran en la Tabla I.

Simultáneamente a estas medidas un vibrómetro láser permite efectuar la medida de la velocidad de un punto del resonador y obtener la dependencia de la velocidad $v$ (en modulo y fase) con la amplitud de la excitación $I_{1}$ (Fig 9). Experimentalmente se observa una débil dependencia del cociente entre la parte mecánica del desplazamiento eléctrico y la deformación elástica $D_{1} / S$ con la velocidad $v$.

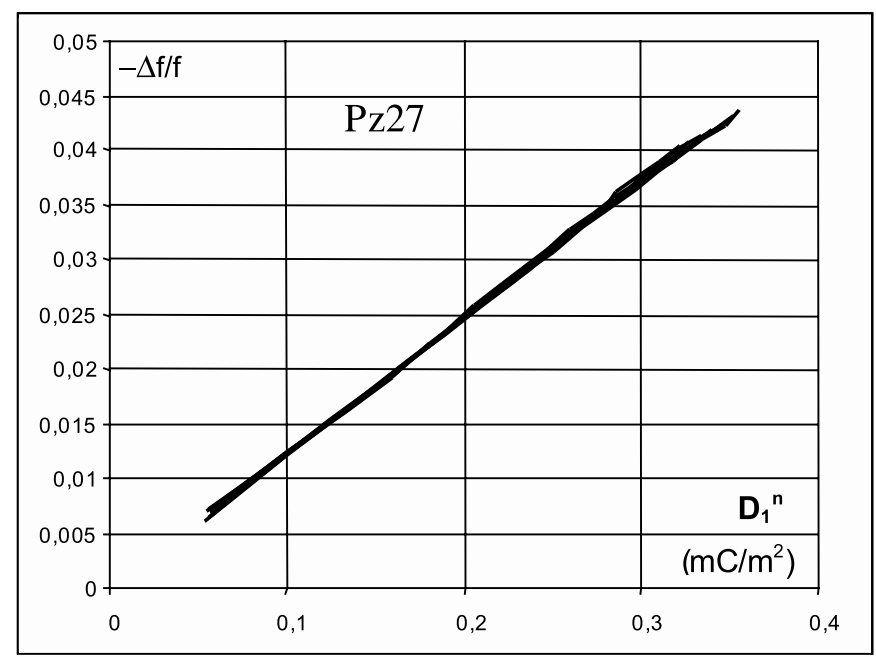

Fig. 8: Variación no lineal de frecuencia en función de una función potencial de la parte mecánica de la corriente de desplazamiento, elevada a una potencia no entera $n$.

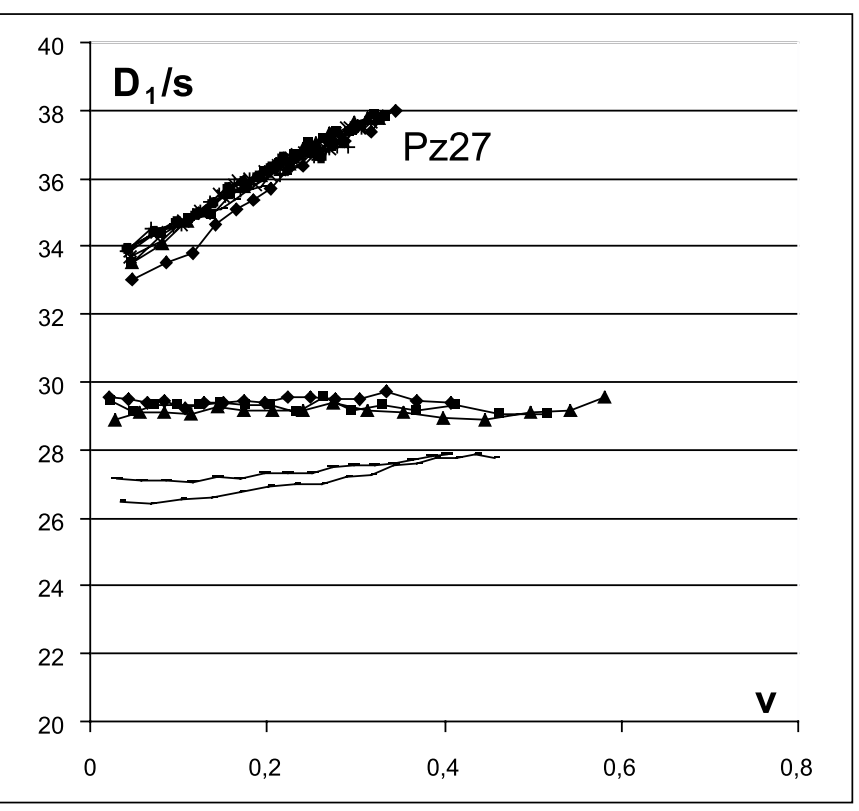

Fig. 9: Variación no lineal del cociente entre la parte mecánica del desplazamiento eléctrico $D_{1}$ y la deformación elástica $S$, en función de la velocidad de un extremo del resonador, para las dos cerámicas citadas. 


\section{CARACTERIZACIÓN DIELÉCTRICA Y PIEZOELECTRICA}

Las cerámicas piezoeléctricas exhiben no solo anomalías en el comportamiento elástico, sino también en el comportamiento dieléctrico y piezoeléctrico. La medida de dichas anomalías se debe efectuar a frecuencia baja, usualmente $1 \mathrm{kHz}$, notablemente inferior a las frecuencias de resonancia, a fin de no ser perturbadas por estas. Con ello, la aplicación de un campo eléctrico producirá deformaciones en la muestra, que se debe dejar mecánicamente libre para que se pueda expandir sin esfuerzo. El campo aplicado d eberá ser mucho mayor que en el caso de resonancia, y en la medida en que su valor se asemeje al valor del campo coercitivo, se producirá una rápida degradación de la muestra y por último su despolarización.

Si se mide el desplazamiento de los bordes de la muestra, mediante un interferómetro láser, y se relaciona con el campo aplicado, se puede obtener el valor del coeficiente piezoeléctrico $d$ inverso, que será dependiente de la amplitud del campo aplicado.

Si se mide la corriente que circula por la muestra, se puede conocer el desplazamiento eléctrico $D$ y con ello medir la constante dieléctrica. A fin de medir el comportamiento dieléctrico se utiliza un puente de impedancias mediante el cual podemos obtener directamente la corriente no lineal, llamando así a la diferencia entre la que corresponde al comportamiento real y al comportamiento lineal (16). Esto nos permite medir la no linealidad incluso cuando su importancia relativa es muy pequeña. También nos permite medir con precisión el factor de pérdidas. Con ello se obtiene los valores de $\varepsilon^{\prime}$ y $\varepsilon^{\prime \prime}$ para cada valor de la amplitud del campo aplicado (Fig. 10). Las medidas se realizan utilizando un tren de 5 ondas de $1 \mathrm{kHz}$, que se repite cada $10 \mathrm{~Hz}$, para evitar el sobrecalentamiento del material.

Analizando los resultados obtenidos en los materiales blandos, se observa que la dependencia de las partes real e imaginaria de la constante dieléctrica sufren incrementos casi proporcionales a la amplitud del campo aplicado, de forma que la relación entre dichos incrementos, puesta de manifiesto en la grafica 11, se obtiene un valor del orden de 0,5 . Ambos hechos están en total coherencia con las predicciones obtenidas de la aplicación del modelo de Rayleigh $(17,18)$ :

$$
\frac{D}{\varepsilon_{0}}=\left(\varepsilon_{0}{ }^{\prime}+\alpha E_{0}\right) E \pm \frac{\alpha}{2}\left(E_{0}^{2}-E^{2}\right)
$$

Comparando dicho resultado con el incremento de impedancia "mecánica" en resonancia, observamos que $\Delta \varepsilon^{\prime} / \Delta \varepsilon^{\prime} \cong \Delta X_{N L} / \Delta R_{N L}=m \cong$ 2 , por lo que el comportamiento elástico obedece, tanto en la dependencia proporcional como en el factor de proporcionalidad, al citado modelo (Tabla I).

En cambio, en los materiales duros la dependencia de $\varepsilon^{\prime}$ y $\varepsilon^{\prime \prime}$ con la amplitud del campo ya no es lineal, sino que sigue una dependencia potencial cuyo exponente $\eta$ se acerca a 2 , tal como ocurría con el comportamiento elástico. Como que el exponente es semejante en los dos casos, la relación $\Delta \varepsilon^{\prime \prime}\left(\Delta \varepsilon^{\prime}\right)$ es también lineal (Fig. 11), pero su pendiente es mucho menor que en el caso de las cerámicas blandas. Para el caso de materiales moderadamente duros, también se cumple que $\Delta \varepsilon^{\prime} / \Delta \varepsilon^{\prime \prime} \cong$ $\Delta X_{N L} / \Delta R_{N L^{\prime}}$ aunque en este caso esta relación es algo mayor que 6.

La comparación entre los coeficientes dieléctricos y piezoeléctricos nos revela un hecho aún más llamativo (Fig. 12): para cada uno de los materiales la relación $d\left(\varepsilon^{\prime}\right)$ es lineal (aunque la dependencia de ellos con el campo no lo sea) y la relación $\Delta d / \Delta \varepsilon^{\prime}$ tiene un valor parecido en todas las cerámicas, sean blandas o duras, y parecido al coeficiente $g=d / \varepsilon^{\prime}(19)$.

Ello revela claramente que los fenómenos no lineales que aparecen no son independientes entre sí. El hecho de que para dos cerámicas PZT distintas, a un mismo incremento de polarización eléctrica le corresponda la misma deformación, implica que los efectos no lineales
Pz 27

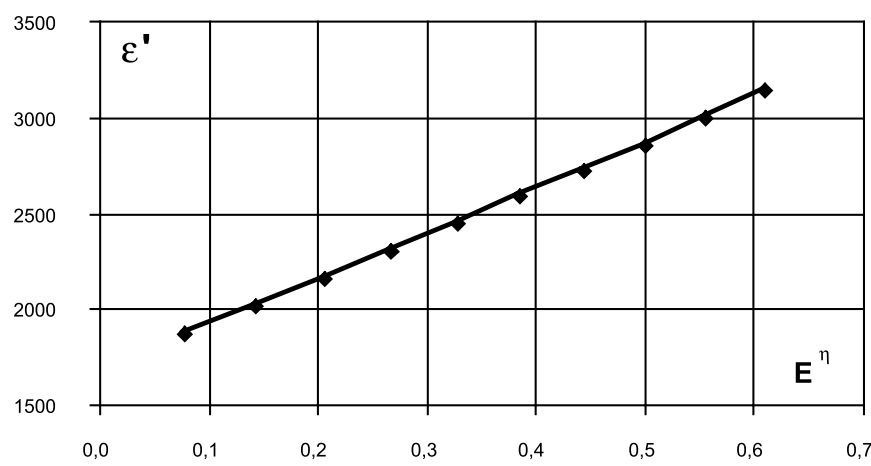

Fig. 10: Dependencia potencial de la constante dieléctrica $\varepsilon^{\prime}$ con la amplitud del campo eléctrico aplicado, elevado a una potencia no entera $\eta$.

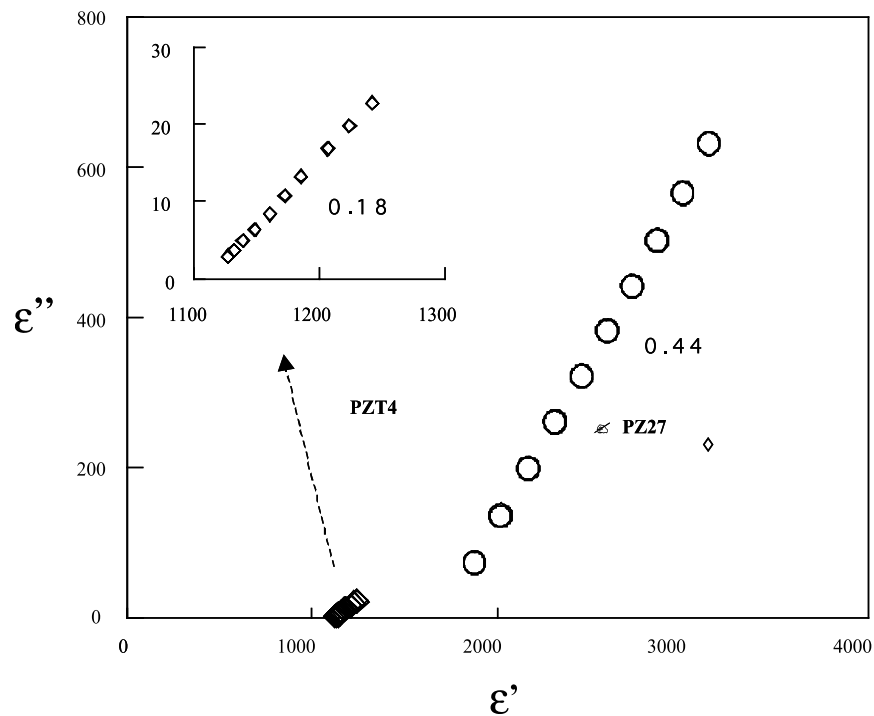

Fig. 11: Variación de las pérdidas dieléctricas $\varepsilon^{\prime \prime}$ en función de la constante dieléctrica $\varepsilon^{\prime}$ para las dos cerámicas citadas.

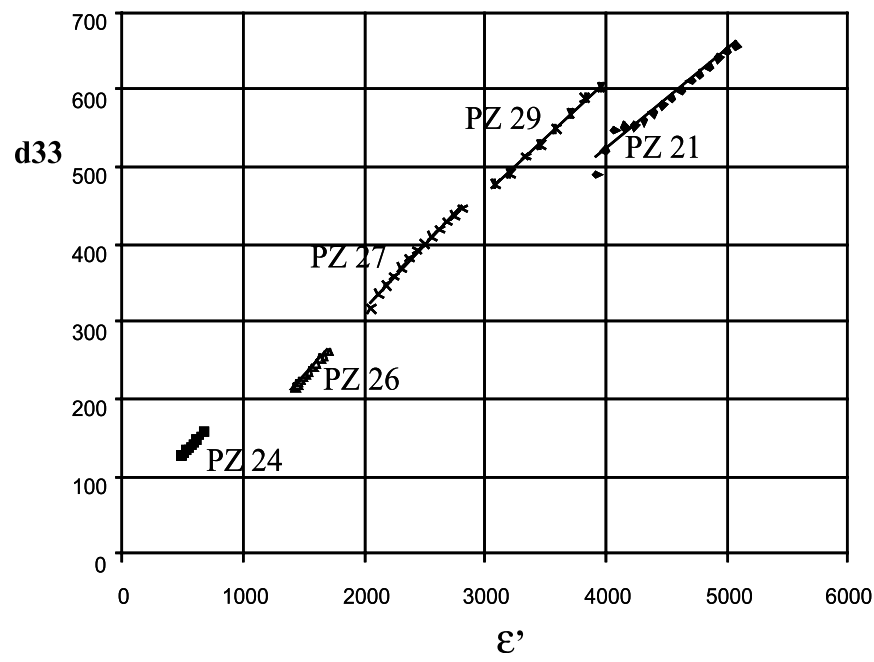

Fig. 12: Relación entre el coeficiente piezoeléctrico $\mathrm{d}_{33} \mathrm{y}$ la constante dieléctrica $\varepsilon^{\prime}{ }_{33^{\prime}}$ para diferentes materiales y diferentes amplitudes.

no se producen por deformación de la celda cristalina (efecto intrínseco), lo que no tiene porque respetar dicha relación, sino que probablemente se produce por movimiento de las paredes (efecto extrínseco) que debe respetarla. 


\section{CONCLUSIONES}

Los tres métodos que pueden usarse para medir la no linealidad en resonancia, ofrecen resultados equivalentes, si bien el método de mantener constante la frecuencia permite obtener de forma más directa y sencilla la relación entre pérdidas y corrimiento de frecuencia con el nivel de excitación.

La mejor forma de conseguir evitar el calentamiento de las muestras cuando se aplica alta señal es la utilización de señales en trenes de onda. En contrapartida el uso de estas señales imposibilita el uso de analizadores de impedancias, precisando obtener las respuestas temporales $I(t)$.

Las medidas experimentales realizadas en la resonancia, donde se obtienen las leyes no lineales elásticas, y las leyes no lineales dieléctricas y piezoeléctricas, muestran grandes similitudes. Probablemente, esta concordancia puede explicarse si se correlacionan las no linealidades con los efectos microscópicos y del movimiento de los dominios.

La concordancia entre las relaciones $\Delta \varepsilon^{\prime} / \Delta \varepsilon^{\prime \prime}$ y $\Delta X_{N L} / \Delta R_{N L^{\prime}}$ a pesar del comportamiento claramente distinto en cuanto a la dependencia con el campo o con la deformación, nos hace suponer que en cada caso, la energía perdida depende de la cantidad de pared desplazada, con independencia de que ello se haya producido por efecto de un campo eléctrico o de la aplicación de un esfuerzo mecánico.

Por último, las diferencias en el valor de esta relación que muestran los distintos tipos de materiales, así como la distinta dependencia con el campo, nos indican la existencia de dos mecanismos distintos, cuyos efectos se presentan en distinta proporción según el material.

\section{AGRADECIMIENTOS}

Este trabajo ha sido posible gracias a la ayuda del MCYT, proyecto ${ }^{\circ}$ MAT2001-2325, así como al apoyo de la red europea POLECER (G5RT-CT-2001-05024).

\section{BIBLIOGRAFÍA}

1. L. E.Cross. "Ferroelectric materials for electromechanical transducer applications" Jpn. J. Appl.Phys., 34, pp 2525-2532, (1995)

2. K. Uchino "Piezoelectric actuators and ultrasonic motors". Kluwer Acad. Pub. 1997.
3. U.Robels, G.Arlt "Domain wall clamping in ferroelectrics by orientation of defects" . J. Appl. Phys. 73, pp 3454-3460, (1993)

4. B.Jaffe, W.Cook, H.Jaffe "Piezoelectric ceramics". Academic Press Ltd. (1971)

5. Q.M. Zhang, H. Wang, N. Kim, L.E.,Cross . "Direct evaluation of domainwall and intrinsic contributions to the dielectric and piezoelectric response and their temperature dependence on lead zirconate-titanate ceramics". J. Appl. Phys., 75, pp 454-459,(1994)

6. S. Li, W. Cao, L.E. Cross ."The extrinsic nature of nonlinear behavior observed in lead zirconate titanate ferroelectric ceramic". J. Appl. Phys., 69, pp 7219-7224, (1991)

7. V. Mueller, Q.M.Zhang "Nonlinearity and scaling behavior in donor-doped lead zirconatre titanate piezoceramic". Appl. Phys. Lett. 72, 2692-2694 (1998)

8. P. Gonnard. "Investigation on dielectric, mechanical and piezoelectric non-linearities in piezoceramics through a new equivalent circuit " . Proc. ISAF'00. pp 691 (2001)

9. European Standard P25EN 50324-3 “Piezoelectric properties of ceramic materials and components - Part 3: Properties and methods of measurement - high power". CENELEC.

10. D. Guyomar, N. Aurelle, C. Richard, P. Gonnard, L.Eyraud "Non Linearities in Langevin Transducers" Proc IEEE Int.Ultrasonics Symposium -Cannes Nov 1994 (1994)

11. R. Pérez, A. Albareda . "Analysis of nonlinear effects in a piezoelectric resonator". J. Acoust. Soc. Am. 100 (6).pp 3561-3569 (1996). Proc. Mater. por Smart Systems. Mater. Res. Soc. Vol. 360, pp. 15-20 (1995).

12. A. Albareda, J-H Kayombo, J.A. Casals, F. Montero de Espinosa "Comportamiento no lineal de piezocomposites en transductores resonantes de potencia". Bol. Soc. Esp. Ceram. V., 38 (5), 510-513 (1999).

13. S. Hirose, S. Takahashi, K. Uchino, M. Aoyagi, Y. Tomikawa. "Measuring methods for high power characteristics of piezoelectric materials".

14. M. Umeda, K. Nakamura, S. Ueha, "The measurement of high power characteristics for a piezoelectric transducer based on the electrical transient response", Jpn. J. Appl. Phys., 37, 5322 (1998).

15. A. Albareda, J.A. Casals, R. Pérez, F. Montero de Espinosa "Nonlinear measurements of piezocomposite transducers with burst excitation". Proc.12th-IEEE ISAF'00, pp 979-982 (2001).

16. J.E. García, R. Pérez, A. Albareda. "High electric field measurement of dielectric constant and losses of ferroelectric ceramics". J. Phys. D: Appl. Phys., 34, pp 3279-3284 (2001).

17. D. A. Hall. "Rayleigh behaviour and the threshold field in ferroelectric ceramics" Ferroelectrics, 223, pp 319-328, (1999)

18. D. Damjanovic. "Stress and frequency dependence of the direct piezoelectric effect in ferroelectric ceramics". J. Appl. Phys., 82, pp 1788-1797 (1997).

19. Andersen, E. Ringgaard, T. Bove, A. Albareda, R. Pérez "Performance of Piezoelectric Ceramic Multilayer Components Based on Hard and Soft PZT". Proc. ACTUATORS'00, pp. 419-422, (2000).

Recibido: 1.2.03

Aceptado: 30.11 .03 